\title{
The augmentation of O-GlcNAcylation reduces glyoxal-induced cell injury by attenuating oxidative stress in human retinal microvascular endothelial cells
}

\author{
GUO DONG LIU ${ }^{1 *}, \mathrm{CHONG} \mathrm{XU}^{1,2^{*}}, \mathrm{LE} \mathrm{FENG}^{1}$ and FANG WANG ${ }^{1}$ \\ ${ }^{1}$ Department of Ophthalmology, Shanghai Tenth People's Hospital Affiliated to Tongji University School of Medicine, \\ Shanghai 200072; ${ }^{2}$ Nanshan Maternity and Child Healthcare Hospital of Shenzhen, Shenzhen 518000, P.R. China
}

Received February 14, 2015; Accepted August 11, 2015

DOI: 10.3892/ijmm.2015.2319

\begin{abstract}
It has recently been reported that O-linked $\beta$-N-acetyl glucosamine (O-GlcNAc) modification (a simple intracellular serine (Ser)/threonine (Thr)-linked monosaccharide) in human retinal microvascular endothelial cells (HRECs) is related to diabetic retinopathy (DR). During O-GlcNAcylation, O-GlcNAc is added to Ser and Thr residues. As the generation of reactive oxygen species (ROS) is one of the characteristics of advanced glycation end product (AGE) injury, and the most important key pathogenic factor of DR, in the present study, we aimed to investigate the association between O-GlcNAcylation and ROS generation in order to ascertain whether O-GlcNAcylation mitigates cellular injury through the generation of ROS. For this purpose, HRECs were divided into 4 groups as follows: HRECs treated with normal glucose $(5 \mathrm{mM})$, HRECs treated with glyoxal $(500 \mu \mathrm{M})$, glyoxal-treated HRECs also treated with $200 \mu \mathrm{M}$ PUGNAc, and glyoxal-treated HRECs infected with O-GlcNAc transferase (OGT) siRNA. We detected increased O-GlcNAc levels and increased ROS production in the glyoxal-treated HRECs. The cellular redox status was determined by cellular ROS staining and by measuring the expression levels of the antioxidant genes, superoxide dismutase (SOD) and glutathione peroxidase (GPX). While the augmentation of O-GlcNAcylation following treatment with PUGNAc significantly attenuated the production of ROS $(\mathrm{P}<0.01)$ and increased the expression levels of SOD and GPX, the reduction of O-GlcNAcylation following infection with OGT siRNA, exacerbated the production of ROS $(\mathrm{P}<0.01)$ and decreased the expression of antioxidant
\end{abstract}

Correspondence to: Dr Fang Wang, Department of Ophthalmology, Shanghai Tenth People's Hospital Affiliated to Tongji University School of Medicine, 301 Middle Yan Chang Road, Shanghai 200072, P.R. China

E-mail: dreyemilwang_122@163.com

${ }^{*}$ Contributed equally

Key words: O-GlcNAcylation, reactive oxygen species, human retinal microvascular endothelial cells, diabetic retinopathy genes. The effects of O-GlcNAcylation on the viability of HRECs were significant $(\mathrm{P}<0.01)$, particularly in the hydrogen peroxide $\left(\mathrm{H}_{2} \mathrm{O}_{2}\right)$-treated HRECs. Treatment with PUGNAc reduced glyoxal-induced cell apoptosis and transfection with OGT siRNA increased HREC apoptosis; these results were confirmed by flow cytometry and by the assessment of mitochondrial membrane potential. The augmentation of O-GlcNAcylation exerted cytoprotective effects on the HRECs by reducing the generation of ROS, increasing the expression of antioxidant genes, preventing the dissipation of mitochondrial membrane potential and preventing HREC apoptosis. Therefore, it can be concluded that O-GlcNAcylation plays a role in the early developmental process of DR.

\section{Introduction}

The prevalence of diabetes mellitus continues to increase worldwide, with diabetic retinopathy (DR) remaining a leading cause of vision loss in several countries. The pathogenesis of DR is multifactorial and affects all cell types in the retina (1). Although hyperglycaemia is recognised as a symptom and a complication of diabetes, the precise molecular mechanisms affected during hyperglycaemic conditions are not yet well understood. In previous studies, glyoxal and methylglyoxal were defined as toxic metabolites and their levels were proven to increase under hyperglycaemic conditions (2). Glyoxal and methylglyoxal promote the formation of advanced glycation end products (AGEs) to produce oxidative stress, and they can cause various types of cellular damage (3). As one factor responsible for the generation of reactive oxygen species (ROS), AGEs have been implicated in the pathogenesis of DR (4-6), cataractogenesis $(7)$ and other diabetic complications $(8,9)$. Under hyperglycaemic conditions, ROS increase vascular permeability and induce retinal ischaemia, which are common pathways in the development and progression of DR $(5,6,10)$. The addition of O-linked $\mathrm{N}$-acetylglucosamine to intracellular proteins (O-GlcNAcylation) is an inducible and a reversible process and it occurs in both the cytoplasm and mitochondria. It involves the post-translational modification of serine (Ser)/threonine (Thr) residues, in the presence of O-GlcNAc transferase (OGT) and O-GlcNAcase (OGA) (11). Studies on several transformed cell lines have reported that high glucose levels increase O-linked 
$\beta$-N-acetyl glucosamine (O-GlcNAc) levels through a number of different mechanisms, which are possibly important in the pathogenesis of diabetes $(12,13)$. O-GlcNAc levels increase in response to hyperglycaemia or glyoxal-induced stress; this mechanism is associated with increased cell survival (14-16). The protective effects of O-GlcNAcylation and the association between O-GlcNAcylation and apoptosis have been previously investigated $(17,18)$. To the best of our knowledge however, there is no study available to date on the association between O-GlcNAcylation and cell apoptosis in DR. Thus, as the association between O-GlcNAcylation and cellular function is complex and poorly understood as regards DR, in the present study, we exposed human retinal microvascular endothelial cells (HRECs) to glyoxal to establish the detrimental effects of ROS and treated them with PUGNAc in order to ascertain whether O-GlcNAcylation prevents cellular injury through its effects on ROS generation.

\section{Materials and methods}

Reagents. The following reagents were purchased: CTD110.6 antibody (sc-59623) was purchased from Covance Inc. (Princeton, NJ, USA); antibodies against caspase-3 (\#9665) and $\beta$-actin (\#8457) were purchased from Cell Signaling Technology (Danvers, MA, USA); anti-von Willebrand factor (vWF; ab6994) was purchased from Abcam (Cambridge, MA, USA); 5,5',6,6'-tetrachloro-1,1',3,3'-tetraethylbenzimidazolyl-carbocyanine iodide (JC-1) and 2',7'dichlorofluorescein diacetate (DCFH-DA) were purchased from the Beyotime Institute of Biotechnology (Haimen, China); PUGNAc, N-acetyl cysteine (NAC) and glyoxal were all obtained from SigmaAldrich (St. Louis, MO, USA); the Annexin V-FITC Apoptosis detection kit was purchased from BD Biosciences (San Diego, CA, USA); fetal bovine serum (FBS) and trypsin-EDTA were purchased from Gibco (Carlsbad, CA, USA); extracellular matrix (ECM), endothelial cell growth supplements (ECGS) (X100) and penicillin/streptomycin were purchased from Sciencell Research Laboratories (Carlsbad, CA, USA); plastic tissue culture flasks were obtained from Costar (Cambridge, MA, USA); and the Immobilon-NC transfer membrane was purchased from Millipore (Billerica, MA, USA); the real-time PCR system was obtained from Promega, (Madison, WI, USA); CCK-8 was purchased from Dojindo Laboratories (Kumamoto, Japan); and a Leica laser scanning microscope from Leica Microsystems (Mannheim, Germany) was used for scanning.

HREC culture. The HRECs were acquired from Cell Systems Corp. (CSC; Kirkland, WA, USA). Using immunofluorescence staining, we confirmed that the cells expressed vWF, which was demonstrated by green particles in the cytoplasm (data not shown); the cells used in the experiments were within 5 passages of authentication. The cells were grown in ECM supplemented with 5\% FBS, ECGS $100 \mathrm{U} / \mathrm{ml}$ and $1 \%$ penicillin/ streptomycin. The cells were incubated at $37^{\circ} \mathrm{C}$ in a $5 \% \mathrm{CO}_{2}$ incubator. The culture medium was replaced every 3 days, and after reaching confluence, the cells were passaged with a $0.25 \%$ solution of trypsin-EDTA. As regards treatment, the HRECs were divided into 4 groups as follows: HRECs treated with normal glucose (5 mM; normal control), HRECs treated with glyoxal (500 $\mu \mathrm{M}$ to produce oxidative stress), glyoxal- treated HRECs also treated with $200 \mu \mathrm{M}$ PUGNAc (to increase levels of O-GlcNAc), and glyoxal-treated HRECs infected with O-GlcNAc transferase (OGT) siRNA (to decrease the levels of O-GlcNAc). After the HRECs were treated with PUGNAc (an inhibitor of OGA) and transfected with siRNA against OGT in serum-free DMEM for $12 \mathrm{~h}$, they were cultured with glyoxal for a further $24 \mathrm{~h}$.

Western blot analysis. Following the appropriate treatment and rinsing with cold phosphate-buffered saline (PBS), the HRECs were scraped into lysis buffer containing a protease inhibitor cocktail. Equal amounts $(30 \mu \mathrm{g})$ of protein from the cell extracts were separated on a $10 \%$ acrylamide gel and subsequently transferred electrophoretically onto polyvinylidene fluoride transfer membranes at $200 \mathrm{~mA}$ for $1.5 \mathrm{~h}$. After blocking in PBS (10 mM Tris- $\mathrm{HCl}$ buffer, $\mathrm{pH} 8.0,150 \mathrm{mM} \mathrm{NaCl}$ ) containing $5 \%(\mathrm{w} / \mathrm{v}) \mathrm{BSA}$ for $1 \mathrm{~h}$ at room temperature, the membranes were incubated overnight at $4^{\circ} \mathrm{C}$ with primary rabbit monoclonal antibody against anti-O-GlcNAc (CTD110.6; Covance) and anti- $\beta$ actin antibody (Cell Signaling Technology) (all antibodies were used at a 1:1,000 dilution). The blots were washed with PBS-T (0.1\% Tween-20 in PBS) 3 times prior to incubation with the secondary antibody (horseradish peroxidase-conjugated goat anti-mouse IgG, 1:5,000; Pierce Biotechnology, Rockford, IL, USA) for $1 \mathrm{~h}$ at room temperature. The hybridised membrane was washed in PBS-T buffer and visualised using Odyssey (LI-COR Biosciences, Lincoln, NE, USA). The optical density of each band was determined by Quantity One software (Bio-Rad).

siRNA transfection and treatment with PUGNAc followed by treatment wth glyoxal. Total protein O-GlcNAcylation was inhibited with the use of siRNA tarteging OGT (OGT siRNA) and was enhanced with the use of PUGNAc. siRNA targeting OGT was purchased from Shanghai GenePharma Co., Ltd., (Shanghai, China) as previously described (13). The HRECs were seeded at $5 \times 10^{5}$ in 6-well plates in ECM and incubated with Lipofectamine 2000 (Invitrogen, Carlsbad, CA, USA) and siRNA in $0.5 \mathrm{ml}$ of serum-free medium for $20 \mathrm{~min}$. The siRNALipofectamine 2000 complex was added to the cells in $1.5 \mathrm{ml}$ of serum-free medium and maintained for $6 \mathrm{~h}$. Another cell group was incubated with $200 \mu \mathrm{M}$ PUGNAc for $12 \mathrm{~h}$ without serum. All the treated cells were then maintained for $24 \mathrm{~h}$, co-cultured with $500 \mu \mathrm{M}$ glyoxal. On the $3 \mathrm{rd}$ day, the cells were prepared for the assessment of ROS production, and for use in western blot analysis, reverse transcription-quantitative polymerase chain reaction (RT-qPCR), CCK-8 assay and JC-1 staining.

Assessment of ROS production. Changes in intracellular ROS levels were determined by measuring the oxidative conversion of cell permeable DCFH-DA to green fluorescent $2^{\prime}, 7^{\prime}$ dichlorofluorescein (DCF). DCFH-DA is widely used to detect the generation of ROS and to assess overall oxidative stress in toxicological phenomena. DCFH-DA is able to diffuse through the cell membrane, and it is enzymatically hydrolysed by intracellular esterases to produce non-fluorescent DCFH. To evaluate the generation of ROS, DCFH-DA dissolved in DMSO was added to the cell cultures at a final concentration of $5 \mu \mathrm{M}$, for $20 \mathrm{~min}$. The cells were lysed with $400 \mathrm{mM} \mathrm{NaOH}$. The total fluorescence intensity of each well was quantified using a fluorescence multi-well plate reader (Synergy 2; BioTek 
Instruments, Inc., Winooski, VT, USA) with excitation and emission wavelengths of 485 and $530 \mathrm{~nm}$, respectively. Total protein concentrations were determined using bicinchoninic acid (BCA) protein assay kits (Pierce Biotechnology). For quantification, ROS levels were assessed by determining the fluorescence intensity/protein concentration. ROS levels were also assessed using fluorescence microscopy to observe the changes in DCF fluorescence.

Cell viability assay. The HRECs were seeded in 96-well plates (3,000 cells/well) and grown for $24 \mathrm{~h}$ in medium supplemented with $5 \% \mathrm{FBS}$, at $37^{\circ} \mathrm{C}$ in $5 \% \mathrm{CO}_{2}$. The cells were then treated as described above. In order to confirm that the damage from ROS was induced by glyoxal, before co-culturing with $500 \mu \mathrm{M}$ glyoxal, one group of cells was treated with NAC $(5 \mathrm{mM})$. Subsequently, $10 \mu 1$ of CCK-8 solution \{containing WST-8 [2-(2-methoxy-4-nitrophenyl)-3-(4-nitrophenyl)-5-(2,4-disulfophenyl)-2H-tetrazolium, monosodium salt]\} was added to each well of the plate (total medium $100 \mu \mathrm{l} /$ well) and the cells were incubated at $37^{\circ} \mathrm{C}$. After $4 \mathrm{~h}$ of incubation, the absorbance was measured using a multi-well plate reader at a wavelength of 450 (650 $\mathrm{nm}$ reference wavelength). The effects of O-GlcNAcylation on oxidative stress-induced damage to the HRECs were then determined. In order to confirm that O-GlcNAcylation protects the HRECs from releasing ROS, the treated HRECs (siRNA transfection, treatment with PUGNAc and NAC) were exposed to $200 \mu \mathrm{M}$ hydrogen peroxide $\left(\mathrm{H}_{2} \mathrm{O}_{2}\right)$ for $2 \mathrm{~h}$; the viability of the HRECs was then assessed by CCK-8 assay as already described above.

$R T-q P C R$ for the measurement of superoxide dismutase (SOD) and glutathione peroxidase (GPX) mRNA expression. Total RNA was extracted using TRIzol reagent (Invitrogen). The synthesis of the cDNA was performed using $1 \mu \mathrm{g}$ of total RNA with reverse transcription reagents (Takara Bio, Inc., Shiga, Japan) and qPCR was carried out on the Bio-Rad iQ5 Optical System in $20 \mu \mathrm{l}$ TaqMan Gene Expression Master Mix (Promega) using $200 \mathrm{ng}$ cDNA. Human primer sets were ordered and used according to the manufacturer's instructions. The human $\beta$-actin gene was used as an endogenous reference to control for the independent expression of sample-to-sample variability. The relative expression of the target genes was normalised by dividing the target $\mathrm{Ct}$ value by the endogenous $\mathrm{Ct}$ values. The primer sequences that were tested in the present study were as follows: human SOD sense, 5'-GCAATGTGA CTGCTGACAAAGAT-3' and antisense, 5'-ATTACACCA CAAGCCAAACGACT-3'; human GPX sense, 5'-ACTCT CTCGTTTCCTTTCTGTTGCT-3' and antisense, 5'-CTCTT CGTTCTTGGCGTTCTCC-3'; and human $\beta$-actin sense, 5'-ATGTCACGCACGATTTCCC-3' and antisense, 5'-GAGA CCTTCAACACCCCAGC-3'.

Annexin Vand PIdouble staining byflow cytometry. The HRECs were incubated with $200 \mu \mathrm{M}$ PUGNAc or infected with siRNA for $12 \mathrm{~h}$ without serum. All the treated cells were then cultured for $24 \mathrm{~h}$ with $500 \mu \mathrm{M}$ glyoxal. As the positive control, the cells treated with PUGNAc and infected with siRNA were incubated with $\mathrm{H}_{2} \mathrm{O}_{2}(100 \mu \mathrm{M})$ for $12 \mathrm{~h}$. The cells were resuspended in Annexin $\mathrm{V}$ binding buffer (BD Biosciences) at a concentration of $1 \times 10^{6}$ cells $/ \mathrm{ml}$. Annexin V-FITC (BD Biosciences) was then added followed by incubation for $15 \mathrm{~min}$ in the dark in a $100 \mu \mathrm{l}$ cell suspension. PI was then spiked into $400 \mu \mathrm{l}$ Annexin V binding buffer and added immediately to the cell suspension, and subsequently analysed on a FACScan flow cytometer (Becton-Dickinson, Franklin Lakes, NJ, USA).

Assessment of mitochondrial membrane potential. Mitochondrial membrane potential was examined by staining with JC-1, a lipophilic, cationic dye that exhibits a fluorescence emission shift upon aggregation from $530 \mathrm{~nm}$ (green) to $590 \mathrm{~nm}$ (red). In healthy cells with a high mitochondrial membrane potential, JC-1 enters the mitochondrial matrix in a potentialdependent manner and forms aggregates. Staining was performed using $2.5 \mu \mathrm{M} \mathrm{JC}-1$ at $37^{\circ} \mathrm{C}$ for $15 \mathrm{~min}$. Following staining, the cells were rinsed 3 times with PBS. Images were captured using an inverted fluorescence microscope (Leica Microsystems) at excitation/emission wavelengths of $530 / 590 \mathrm{~nm}$. The fluorescence intensity was analysed using Image-Pro Plus v6.0 image analysis software.

Statistical analysis. All experiments were performed with 3 biological replicates. The statistical signifiance of the differences was determined using one-way analysis of variance followed by Dunnett's multiple comparison test. Values are presented as the means \pm standard deviation and a value of $\mathrm{P}<0.05$ was considered to indicate a statistically significant difference.

\section{Results}

O-GlcNAcylationand caspase-3levels inHRECs. The totallevels of O-GlcNAc were determined by western blot analysis using CTD110.6, as described in the Materials and methods (Fig. 1A). The O-GlcNAc levels increased in the glyoxal-treated HRECs compared with the normal glucose-treated HRECs (Fig. 1B, $\mathrm{P}<0.05)$. PUGNAc, as an OGA inhibitor, significantly increased the O-GlcNAc levels (Fig. 1B, P<0.01), while transfection with OGT siRNA decreased the levels of O-GlcNAc in the presence of glyoxal (Fig. 1B, P<0.01). Our results also demonstrated that the expression level of total caspase- 3 decreased in the presence of glyoxal. The augmentation of O-GlcNAcylation increased the expression of total caspase-3, whereas the attenuation of O-GlcNAcylation (by siRNA) decreased the expression of total caspase-3 (Fig. 1C, $\mathrm{P}<0.01$ ). These results clearly indicated that glyoxal increased the total levels of O-GlcNAc and that the augmentation of O-GlcNAcylation protected the HRECs from glyoxal-induced damage. Cleaved caspase-3 is a critical executioner of apoptosis. The activation of caspase- 3 requires the proteolysis of total caspase- 3 into cleaved caspase- 3 . The level of total caspase- 3 increased, which indicates that the level of cleaved caspase- 3 decreased $(19,37,38)$. The augmentation of O-GlcNAcylation increased the level of total caspase- 3 and decreased the proteolysis of total caspase-3, which indicated that the augmentation of O-GlcNAcylation reduced the level of cleaved caspase- 3 and cell apoptosis; thus, our results suggest that the augmentation of O-GlcNAcylation protects the HRECs from glyoxal-induced damage.

Effects of O-GlcNAcylation on glyoxal-induced ROS generation. ROS are important contributors to AGE-induced injury 
A

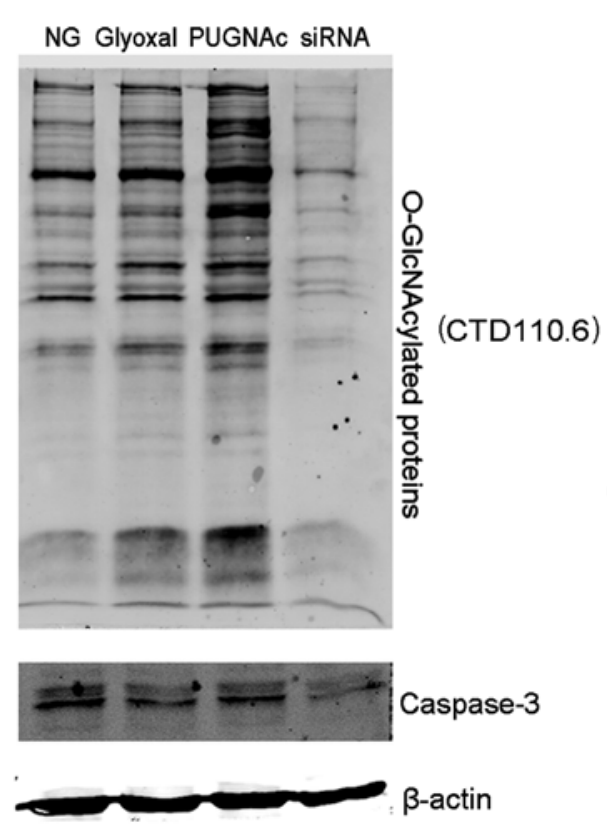

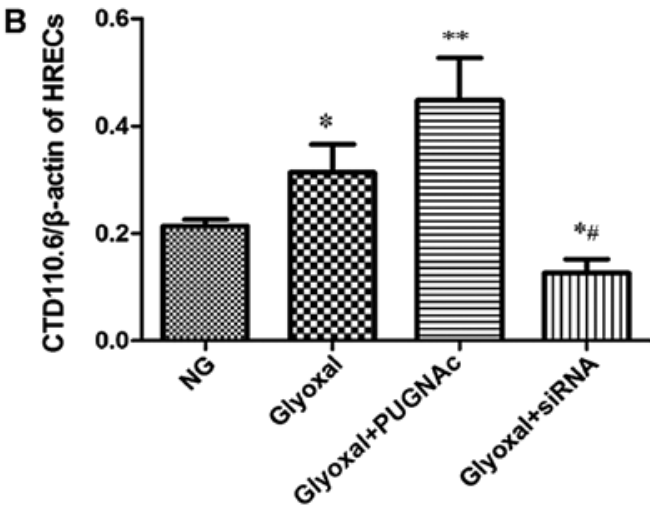

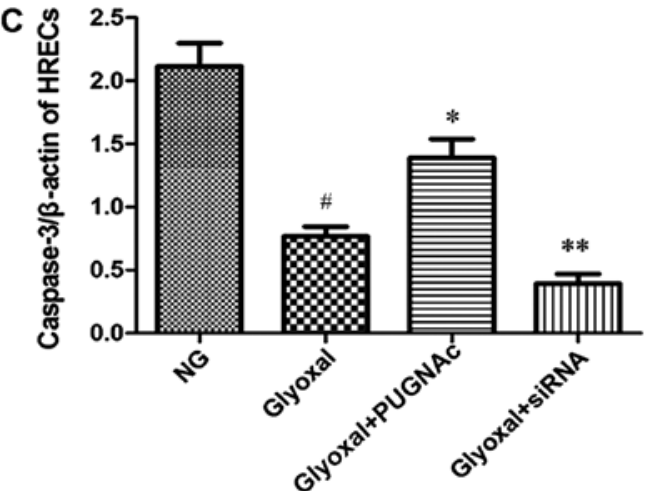

Figure 1. Levels of O-GlcNAc and caspase-3 in human retinal microvascular endothelial cells (HRECs). (A) Western blot analysis; the levels of O-GlcNAc in each lane and the levels of total caspase-3 were quantified using Quantity One software. (B) Treatment of HRECs with glyoxal for $24 \mathrm{~h}$ induced an increase in O-GlcNAcylation. "P $<0.05$ vs. NG. PUGNAc increased O-GlcNAcylation by the inhibition of OGA. ${ }^{* *} \mathrm{P}<0.01$ vs. glyoxal. OGT siRNA decreased O-GlcNAcylation. ${ }^{* *} \mathrm{P}<0.01$ vs. glyoxal. Data are representative of results from 1 of 3 independent experiments with similar results. (C) The expression of total caspase-3 was examined by western blot analysis. In the presence of glyoxal, the expression of total caspase- 3 decreased, ${ }^{,} \mathrm{P}<0.05$. The fffects of $\mathrm{O}-\mathrm{GlcNA}$. $y l a t i o n$ on caspase-3 epxression were examined by western blot analysis. While the augmentation of O-GlcNAcylation increased total caspase-3 expression, decreased O-GlcNAcylation also decreased total caspase- 3 expression ${ }^{* *} \mathrm{P}<0.01$, vs. glyoxal. NG, normal glucose.
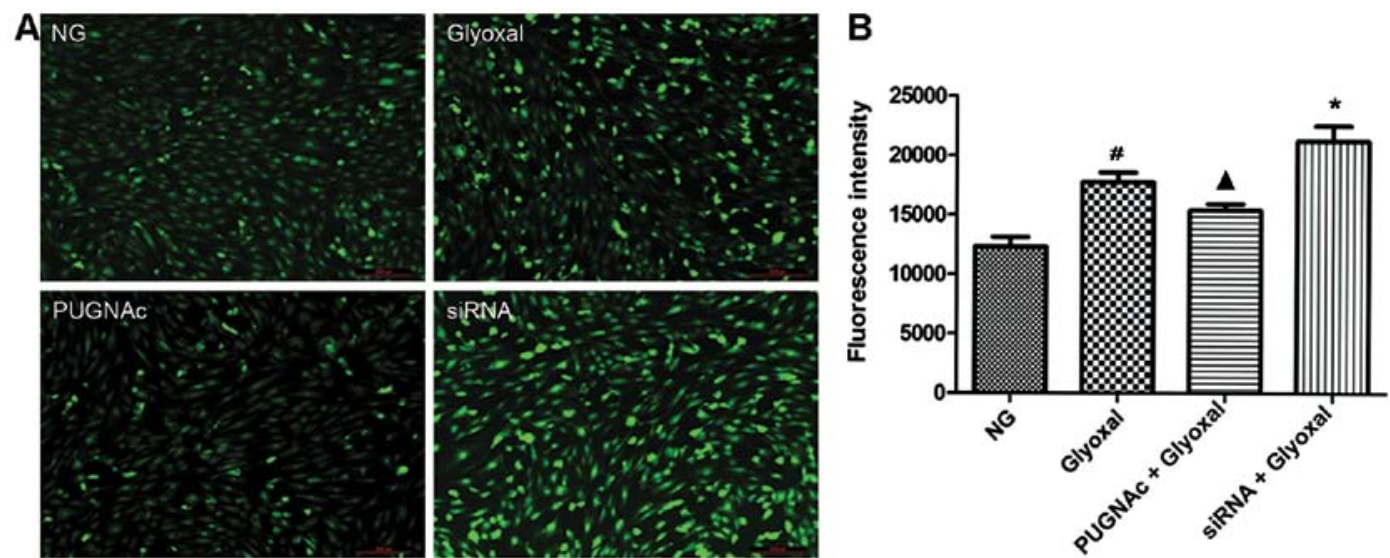

Figure 2. Effects of O-GlcNAcylation on glyoxal-induced reactive oxygen species (ROS) generation. Human retinal microvascular endothelial cells (HRECs) were treated with PUGNAc $(200 \mu \mathrm{M})$ or OGT siRNA and they were then exposed to glyoxal $(500 \mu \mathrm{M})$. ROS levels were measured using DCF (n=3 per group). (A) DCFH-DA staining for ROS generation of HRECs in the presence of glyoxal, PUGNAc or OGT siRNA using fluorescence microscopy. (B) Quantitative changes in mean DCF fluorescence in HRECs exposed to glyoxal, PUGNAc or OGT siRNA using an automatic microplate reader. While PUGNAc significantly attenuated ROS generation induced by glyoxal, OGT siRNA significantly increased ROS generation. ${ }^{*} \mathrm{P}<0.01$, glyoxal vs. NG; ${ }^{\wedge} \mathrm{P}<0.05$, glyoxal $+\mathrm{PUGNAc}$ vs. glyoxal; "P<0.05, glyoxal + siRNA vs. glyoxal. NG, normal glucose.

and they are important in the development of DR (20-23). Images captured by fluorescence microscopy confirmed a higher level of ROS in vitro in the presence of glyoxal, as shown by the strong bright green fluorescence in the representative image (Fig. 2A). Our data confirmed that the ROS levels were higher in the glyoxal-treated HRECs compared with the normal glucose-treated HRECs (Fig. 2B, $\mathrm{P}<0.01$ ). Both the inhibition of OGT (by PUGNAc) and OGA (by siRNA) significantly altered the ROS levels. Glyoxal-induced ROS production was attenuated by increased O-GlcNAcylation $(\mathrm{P}<0.05)$, while OGT siRNA increased the generation of $\operatorname{ROS}(\mathrm{P}<0.05)$. These data indicated that the 

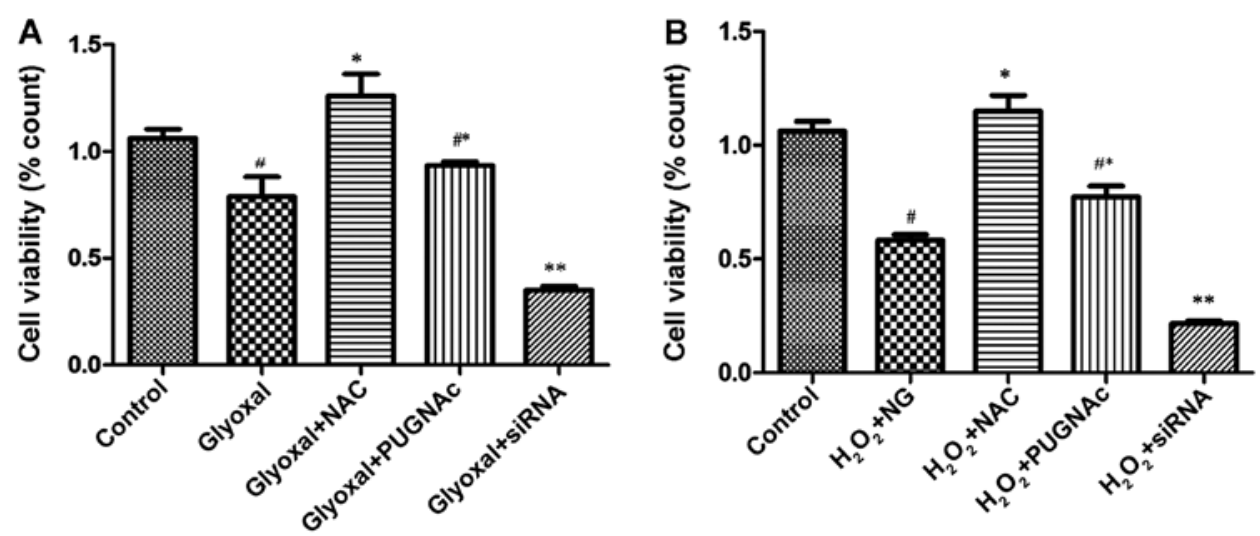

Figure 3. Effects of O-GlcNAcylation on the viability of human retinal microvascular endothelial cells (HRECs). (A) HRECs were transfected with OGT siRNA and treated with PUGNAc $(200 \mu \mathrm{M})$ for $12 \mathrm{~h}$. Cell viability was assessed by CCK-8 assay after the cells were exposed to glyoxal (500 $\mu \mathrm{M})$ for $24 \mathrm{~h}$. Compared with the control group (normal glucose), the cell viability of the glyoxal group was significantly lower $\left({ }^{*} \mathrm{P}<0.005\right.$ vs. control). The viability of the PUGNActreated cells was higher compared with the glyoxal group, although the difference was not significant (\#"P>0.05 vs. glyoxal); however, the OGT siRNA-treated group showed a significant decrease in viability $\left({ }^{* *} \mathrm{P}<0.0001 \mathrm{vs}\right.$. glyoxal). The cell viability of the glyoxal group treated with NAC increased significantly ("P<0.001 vs. glyoxal). (B) Treated HRECs were incubated with $\mathrm{H}_{2} \mathrm{O}_{2}(200 \mu \mathrm{M})$ following treatment with glyoxal, for $2 \mathrm{~h}$. Cell viability was determined by CCK-8 assay. The viability of the cells treated with PUGNAc or OGT siRNA changed significantly $\left({ }^{* *} \mathrm{P}<0.001\right.$ vs. $\mathrm{H}_{2} \mathrm{O}_{2}+\mathrm{NG}$. ${ }^{* *} \mathrm{P}<0.0001$ vs. $\left.\mathrm{H}_{2} \mathrm{O}_{2}+\mathrm{NG}\right)$. As a positive control, the viability of the cells treated with NAC increased significantly ${ }^{*} \mathrm{P}<0.0001$ vs. $\mathrm{H}_{2} \mathrm{O}_{2}+\mathrm{NG}$. NG, normal glucose.
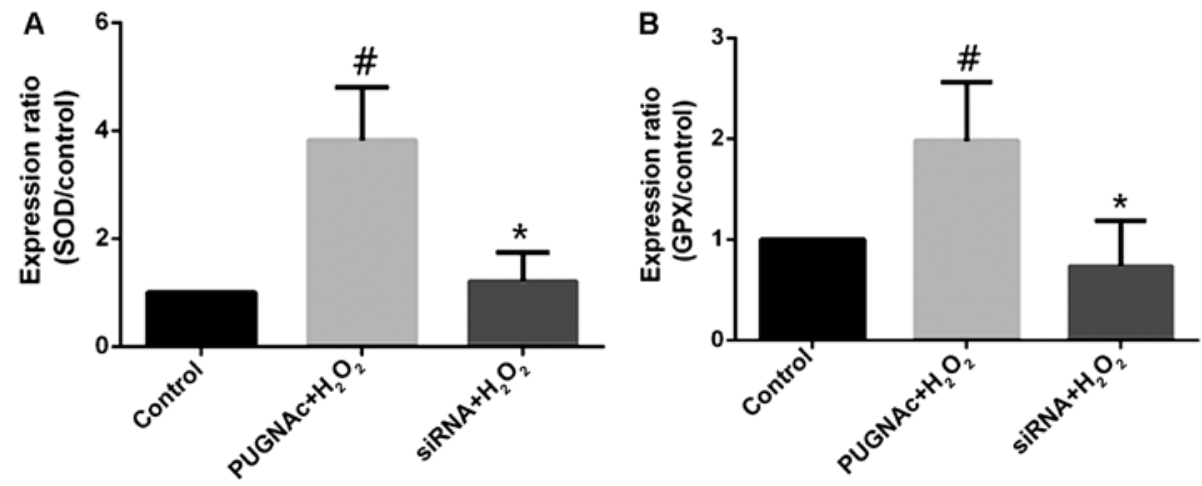

Figure 4. RT-qPCR was used to determine the effects of O-GlcNAcylation on $\mathrm{H}_{2} \mathrm{O}_{2}$-induced alterations in antioxidant gene expression in human retinal microvascular endothelial cells (HRECs). Total RNA was harvested from HRECs and it was converted to single-stranded cDNA and used as template in qPCR with primers hybridizing to the indicated genes (SOD, GPX). (A) Inhibition of OGA with PUGNAc increased superoxide dismutase (SOD) $\mathrm{mRNA}$ levels ( $\mathrm{P}>0.05$ ), while OGT siRNA decreased SOD mRNA levels ("P>0.05). (B) Differences in glutathione peroxidase (GPX) expression between treated and control cells (normal glucose) were insignificant at ${ }^{\#} \mathrm{P}>0.05$. " $\mathrm{P}>0.05$ vs. NG. The trend observed in the changes in epxression suggests that PUGNAc increased GPX mRNA levels, while OGT siRNA decreased GPX mRNA levels. NG, normal glucose.

augmentation of O-GlcNAcylation decreased the oxidative stress induced by glyoxal.

Effects of O-GlcNAcylation on the viability of HRECs. We examined the effects of O-GlcNAcylation on the viability of HRECs exposed to the different treatments. Our statistical analysis revealed that there were significant differences between some of the treatment groups. The cell viability of the glyoxaltreated group was significantly lower compared with that of the control group (normal glucose; $\mathrm{P}<0.005$ ) (Fig. 3A). The viability of the PUGNAc-treated cells was higher compared with that of the glyoxal group, although the difference was not significant $(\mathrm{P}>0.05)$; however, the viability of the OGT siRNA-treated group was significantly lower $(\mathrm{P}<0.0001)$. Following treatment with NAC, the viability of the glyoxal-treated group increased significantly, indicating that O-GlcNAcylation protected the cells by attenuating oxidative stress. To confirm that the reduction of oxidative stress was a contributory mechanism involved in the protective effects of O-GlcNAcylation, the treated HRECs were exposed to $\mathrm{H}_{2} \mathrm{O}_{2}(200 \mu \mathrm{M})$ for $2 \mathrm{~h}$. The viability of the HRECs was then assessed. As shown in Fig. 3B, the decrease in cell viability induced by $\mathrm{H}_{2} \mathrm{O}_{2}$ was mitigated by the OGA inhibitor (PUGNAc) and aggravated by OGT siRNA (Fig. 3B, $\mathrm{P}<0.0001)$. These findings indicated that $\mathrm{O}-\mathrm{GlcNAcylation}$ was associated with changes in cell viability. O-GlcNAcylation reduced the vulnerability of the $\mathrm{HRECs}$ to $\mathrm{H}_{2} \mathrm{O}_{2}$, and protected the cells by attenuating oxidative stress.

Effects of O-GlcNAcylation on the levels of antioxidant enzymes. The HRECs treated with PUGNAc or OGT siRNA were subjected to oxidative stress (with $\mathrm{H}_{2} \mathrm{O}_{2}$ ), and the mRNA levels of antioxidant enzymes were measured by RT-qPCR. The inhibition of OGA with PUGNAc increased the SOD mRNA levels (Fig. 4A, P<0.05), while transfection with OGT siRNA decreased the SOD mRNA levels (not significant compared to the normal glucose-treated group, $\mathrm{P}>0.05$; Fig. 4A). Although 

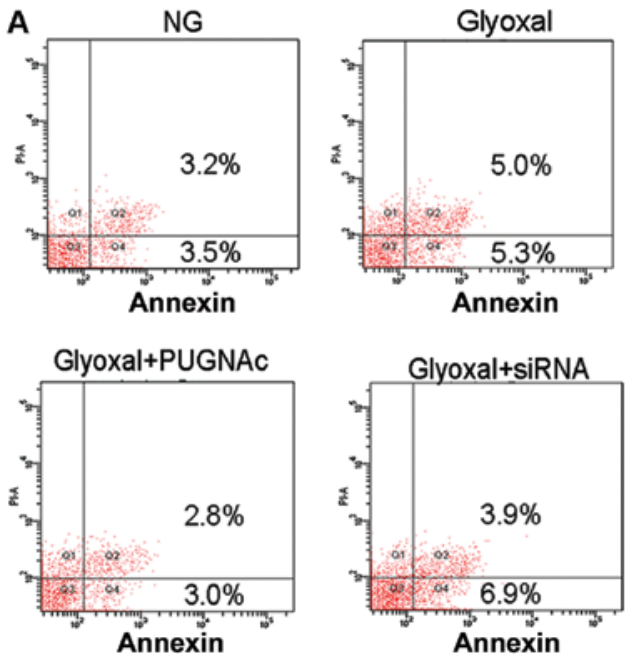

B

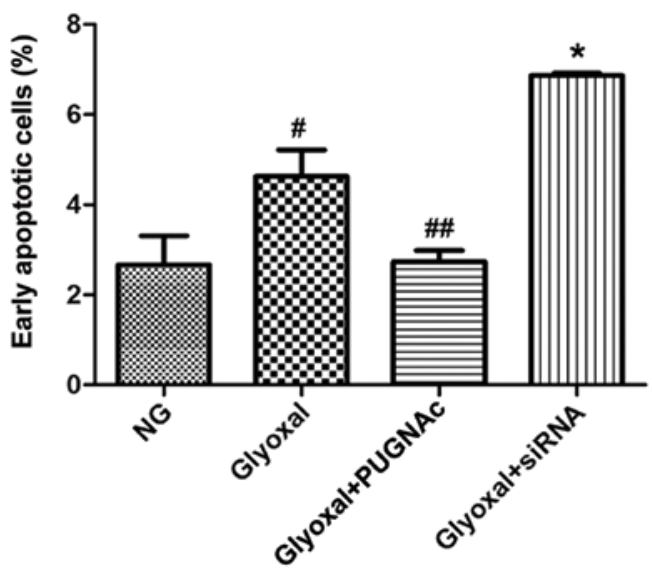

Figure 5. Annexin V and PI double staining by flow cytometry. The bottom right quadrant represents Annexin V-FITC-stained cells (early-phase apoptotic cells) and the top right quadrant represents PI- and Annexin V-FITC-dual-stained cells (late-phase apoptotic/necrotic cells). Apoptotic cells are represented as the percentage of Annexin V single-positive plus Annexin V/PI double-positive cells. O-GlcNAcylation protected the cells from ROS-induced injury caused by glyoxal in human retinal microvascular endothelial cells (HRECs). (A) HRECs were transfected with OGT siRNA and treated with PUGNAc (200 $\mu$ M), and then cultured with glyoxal for $24 \mathrm{~h}$. Cells were stained with Annexin V-FITC and PI and analysed by FACS. (B) Quantification of early apoptosis in HRECs was determined by flow cytometry. While glyoxal treatment alone led to increased apoptosis ( ${ }^{\prime \prime} \mathrm{P}<0.005 \mathrm{vs}$. NG), the combination of glyoxal and PUGNAc resulted in fewer apoptotic cells than those observed with glyoxal alone $\left({ }^{\# \#} \mathrm{P}<0.001\right.$ vs. glyoxal). Downregulation of protein O-GlcNAcylation increased the apoptotic rate of the HRECs significantly ( $\mathrm{P}<0.001$ vs. glyoxal).

changes in GPX mRNA levels occurred in the presence of PUGNAc or OGT siRNA, these changes were not significant compared to the normal glucose-treated group. The trend indicated however, that PUGNAc increased the GPX mRNA levels, while OGT siRNA decreased the GPX mRNA levels (Fig. 4B, $\mathrm{P}>0.05)$.

Effects of O-GlcNAcylation on the apoptosis of HRECs. $\mathrm{O}-\mathrm{GlcN} A c y l a t i o n$ protected the cells by attenuating oxidative stress; thus, the effects of O-GlcNAcylation on the apoptosis of HRECs were also investigated. Treatment with normal glucose resulted in $3.5 \%$ apoptotic cells, which increased to $5.3 \%$ following treatment with glyoxal (Fig.5, $\mathrm{P}<0.005$ ). While glyoxal treatment alone led to apoptosis, the combination of glyoxal and PUGNAc resulted in fewer apoptotic cells compared with glyoxal treatment alone (Fig. 5, $\mathrm{P}<0.001$ ). The decrease in O-GlcNAcylation (by siRNA) significantly increased the apoptotic rate of the HRECs (Fig. 5, P<0.001). Taking these results into account together with the results of western blot analysis, we hypotheiszed that O-GlcNAcylation plays a protective role against cellular apoptosis.

Effects of O-GlcNAcylation on mitochondrial membrane potential. As an indicator of early apoptosis, mitochondrial membrane potential was assessed. The effects of O-GlcNAcylation on mitochondrial membrane potential were determined using a JC-1 probe. As shown in Fig. 6, there was a significant increase in green fluorescence in the cells exposed to glyoxal (Fig. 6, $\mathrm{P}<0.001$ ). Treatment with PUGNAc attenuated the changes in mitochondrial membrane potential induced by glyoxal, as indicated by a decrease in green fluorescence and the restoration of red fluorescence (Fig. 6, P<0.01). However, transfection with siRNA and treatment with glyoxyl abolished the effects of O-GlcNAcylation on mitochondrial membrane potential insignificantly (Fig. 6, P>0.05). These results suggested that the augmentation of O-GlcNAcylation markedly suppressed the glyoxal-induced collapse of mitochondrial membrane potential in HRECs.

\section{Discussion}

DR is a serious complication of diabetes, which can lead to blindness (24). O-GlcNAcylation is important in the pathogenesis of diabetes $(25,26)$, and recent studies have suggested that it participates in the pathogenesis of DR $(12,13)$. Glucose metabolism through the hexosamine biosynthesis pathway (HBP) leads to the formation of uridine 5'-diphosphate-N-acetylglucosamine (UDP-GlcNAc) (8), which serves as the substrate for post-translational modification by OGT and OGA (27,28). ROS, one of the major causes of cellular stress, are associated with the development of DR $(6,29,30)$. During the early stages of DR, high glucose levels lead to retina hypoxia (31), which causes increased intracellular ROS levels. A previous study demonstrated that hypoxia-inducible factor (HIF)-1 $\alpha$ expression levels increased significantly in the vitreous fluid of surgically treated eyes with proliferative DR (32), which also verified the existence of hypoxia, oxidative stress and vascular endothelial growth factor (33). Other studies on O-GlcNAcylation have indicated that when cells are subjected to diverse types of stress (including oxidative stress), the levels of O-GlcNAcylation increase (15). In our previous study on DR, we demonstrated that the levels of O-GlcNAc increased in vitro and in vivo (13). While O-GlcNAcylation has been demonstrated to participate in the process of diabetic complications, the cytoprotective effects have been confirmed in cardiac cells (17). On the other hand, O-GlcNAcylation has also been shown to be part of a mechanism for the regulation of nuclear apoptosis in T cells (18). However, the exact mechanisms responsible for controlling O-GlcNAcylation that occurs in DR remain unclear. In the present study, we aimed 

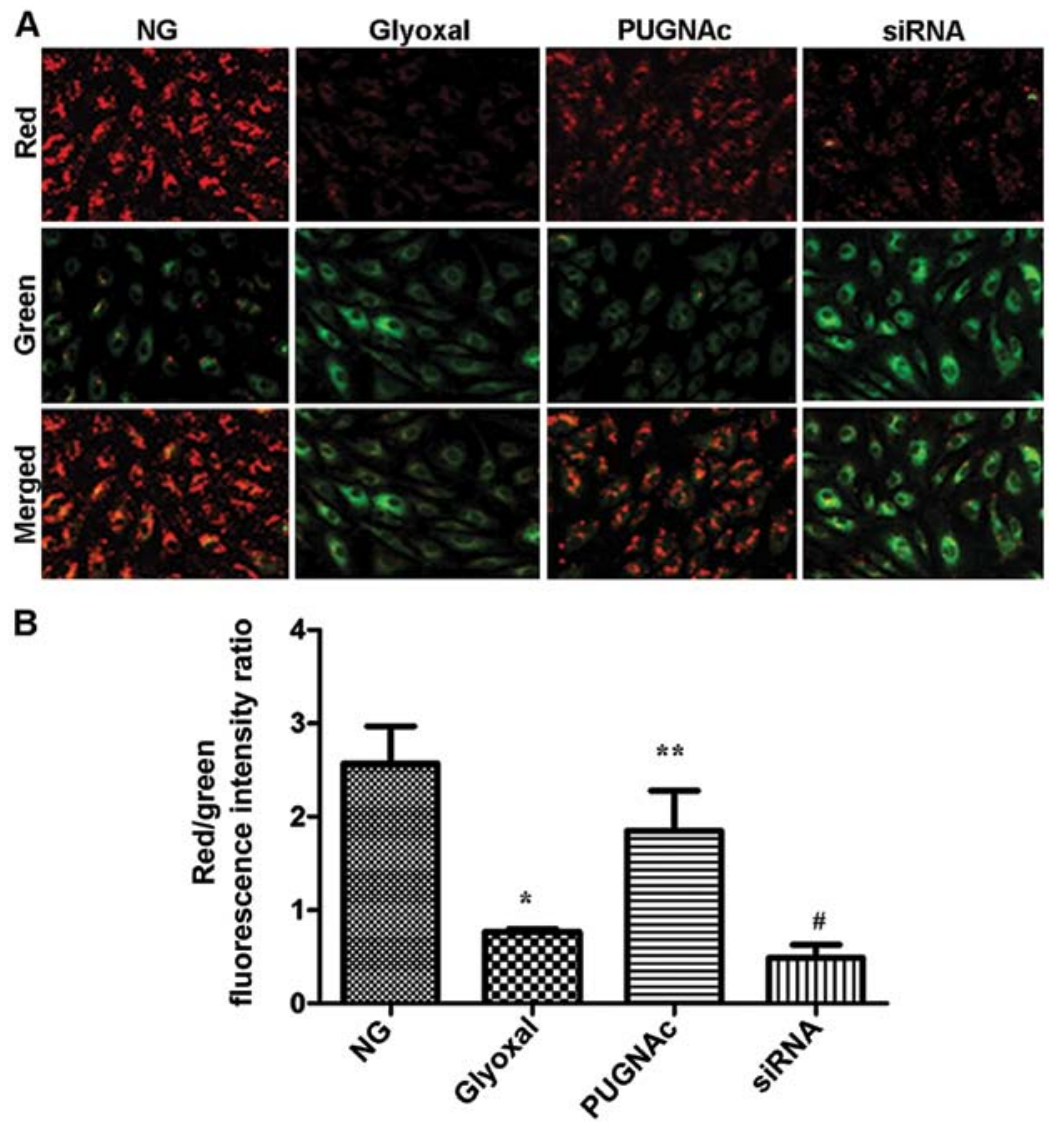

Figure 6. Measurement of mitochondrial membrane potential. Effects of O-GlcNAcylation on mitochondrial membrane potential in human retinal microvascular endothelial cells (HRECs). Cells were treated as described in the Materials and methods and then incubated with the membrane potential indicator, JC-1. (A) Intracellular red and green fluorescence of JC-1 was determined by an inverted fluorescent microscope. The intracellular red and green fluorescence of JC-1 was measured using Image-Pro Plus software. (B) Histogram showing the red/green fluorescence intensity ratio in differently treated cells ( $\mathrm{n}=3$ ). Treatment

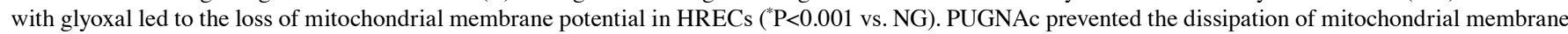
potential, as indicated by the increase in red fluorescence $\left({ }^{* *} \mathrm{P}<0.01\right.$, glyoxal + PUGNAC vs. glyoxal), while siRNA-infected cells showed strong bright green fluorescence $\left({ }^{\#} \mathrm{P}>0.05\right.$, glyoxal + siRNA vs. glyoxal). Attenuation of O-GlcNAcylation insignificantly decreased mitochondrial membrane potential of glyoxal treated cells, but the augmentation of O-GlcNAcylation significantly prevented the dissipation of mitochondrial membrane potential.

to establish a direct association between O-GlcNAcylation and ROS production in glyoxal-damaged HRECs. AGEs or glyoxal-regulated O-GlcNAc modifications increase the apoptosis of HRECs, suggesting that O-GlcNAcylation is an additional mechanism through which cells sense and respond to stress (34). In addition, the mechanisms responsible for increasing O-GlcNAcylation in response to stress may be the result of increasing pools of UDP-GlcNAc induced by glucose uptake (34). Another mechanism of increased O-GlcNAcylation is that oxidative stress activates the $\operatorname{HBP}(35)$, which has been shown to be related to cell protection in some models (36). The reduction of oxidative stress may be a contributory mechanism involved in the protective effects on HRECs, indicating decreased apoptosis and increased cell viability. It is known that the capillaries lined with endothelial cells are responsible for maintaining the blood retinal barrier, and the loss of endothelial cells by apoptosis is one of the most important reasons for the development of DR. While O-GlcNAcylation is involved in the protective effects on HRECs, it may be important in the developmental process of DR.

The results obtained with the fluorescence multi-well plate reader revealed that either augmented (by PUGNAc) or diminished (by OGT siRNA) O-GlcNAcylation significantly altered the baseline ROS levels, induced by glyoxal. Enhanced
O-GlcNAcylation was shown to reduce ROS generation in the presence of glyoxal, while the aggravation of glyoxal-induced ROS generation was observed following transfection with OGT siRNA. Thus, it was concluded that O-GlcNAcylation was one of the regulatory factors that adjusted HREC function by manipulating ROS production. To evaluate the protective effects of O-GlcNAcylation on HRECs, we measured caspase-3 activity, and performed CCK-8 assay, and Annexin V and PI double staining, and measured mitochondrial membrane potential. Total caspase-3 activity was markedly decreased in the HRECs following exposure to glyoxal for $24 \mathrm{~h}$, which indicates that the level of cleaved caspase- 3 had increased $(37,38)$. The level of total caspase- 3 increased when the cells were treated with PUGNAc compared with the group of HRECs treated with glyoxal. However, transfection with OGT siRNA reversed the effects of PUGNAc. We also found that both the augmentation and decrease of O-GlcNAcylation significantly altered cell viability in the presence of glyoxal. Following culture with $\mathrm{H}_{2} \mathrm{O}_{2}$, the protective effects of O-GlcNAcylation on cell viability were more obvious. The changes in cell viability were consistent with the results of early apoptosis in HRECs. Taking these results into account together with the results of western blot anlaysis, we hypothesised that the protective effects of O-GlcNAcylation on HRECs were 
achieved by manipulating ROS production, as shown by decreased ROS generation following treatment with PUGNAc and the aggravation of oxidative stress following transfection with OGT siRNA. Our data confirmed that O-GlcNAcylation attenuated ROS generation by upregulating the activity of the antioxidant enzymes, SOD and GPX. Moreover, as a regulator affecting the transcription of the oxidative stress responsive enzymes, catalase and MnSOD (SOD2), FoxO1 has been shown to be O-GlcNAcylation-modified (39). It is known that the mitochondria are a critical target of O-GlcNAcylationmediated cytoprotection (40), which is associated with ROS production and $\mathrm{Ca}^{2+}$ channels within the mitochondria (41). Nagy et al demonstrated that by enhancing O-GlcNAcylation with glucosamine treatment, $\mathrm{Ca}^{2+}$ overload was blocked in neonatal cardiomyocytes (42). However, the mechanisms through which O-GlcNAcylation attenuates $\mathrm{Ca}^{2+}$ overload under conditions of acute stress remain unknown. It was considered that a mitochondrial OGT isoform existed that interacted with the mitochondrial $\mathrm{Ca}^{2+}$ uniporter, which was related to $\mathrm{Ca}^{2+}$ uptake (43). It has been demonstrated that the augmentation of O-GlcNAcylation attenuates mitochondrial permeability transition pore (mPTP) formation, while diminished O-GlcNAcylation increases mPTP formation (43). These data support our findings demonstrating that enhanced O-GlcNAcylation prevents the collapse of the mitochondrial membrane potential, while diminished O-GlcNAcylation sensitises the cells to the loss of mitochondrial membrane potential. Based on the present data, the destruction of antioxidant enzymes and mitochondrial membrane potential are the reasons for ROS production; therefore, it is possible that O-GlcNAcylation mitigates ROS formation by protecting antioxidant enzymes and maintaining mitochondrial membrane potential. As ROS are involved in cell apoptosis, it can be concluded that O-GlcNAcylation prevents the apoptosis of HRECs by attenuating oxidative stress.

The present study provides strong evidence regarding the possible contribution of increased O-GlcNAcylation to HREC protection, and thus, it can be concluded that the decreased ROS generation is one of the mechanisms through which O-GlcNAcylation reduces the apoptosis of HRECs. Understanding the molecular mechanisms involving O-GlcNAcylation and ROS in HRECs may help to determine the effects of O-GlcNAcylation early on in the development of DR. Thus, regulating O-GlcNAcylation may provide an alternative target in the treatment of DR.

\section{Acknowledgements}

The present study was supported by grants from the National Natural Science Foundation of China Grants (nos. 81271029 and 81300772), the Specialized Research Fund Q2 for the Doctoral Program of Higher Education (20130072120053), and the Science and Technology Commission of Shanghai Q3 (11Jc1409900).

\section{References}

1. Cheung N, Mitchell P and Wong TY: Diabetic retinopathy Lancet 376: 124-136, 2010.

2. Shangari N, Bruce WR, Poon R and O'Brien PJ: Toxicity of glyoxals - role of oxidative stress, metabolic detoxification and thiamine deficiency. Biochem Soc Trans 31: 1390-1393, 2003.
3. Li SY, Sigmon VK, Babcock SA and Ren J: Advanced glycation endproduct induces ROS accumulation, apoptosis, MAP kinase activation and nuclear O-GlcNAcylation in human cardiac myocytes. Life Sci 80: 1051-1056, 2007.

4. Al-Mesallamy HO, Hammad LN,El-Mamoun TA and Khalil BM: Role of advanced glycation end product receptors in the pathogenesis of diabetic retinopathy. J Diabetes Complications 25: 168-174, 2011.

5. Mohamed IN, Soliman SA, Alhusban A, Matragoon S, Pillai BA, Elmarkaby AA and El-Remessy AB: Diabetes exacerbates retinal oxidative stress, inflammation, and microvascular degeneration in spontaneously hypertensive rats. Mol Vis 18: 1457-1466, 2012.

6. Santos JM, Mohammad G, Zhong Q and Kowluru RA: Diabetic retinopathy, superoxide damage and antioxidants. Curr Pharm Biotechnol 12: 352-361, 2011.

7. Scalbert $P$ and Birlouez-Aragon I: Relationship between lens protein glycation and membrane structure in human cataract. Exp Eye Res 56: 335-340, 1993.

8. Buse MG: Hexosamines, insulin resistance, and the complications of diabetes: current status. Am J Physiol Endocrinol Metab 290: E1-E8, 2006.

9. Younessi P and Yoonessi A: Advanced glycation end-products and their receptor-mediated roles: inflammation and oxidative stress. Iran J Med Sci 36: 154-166, 2011.

10. Fernandes R, Hosoya K and Pereira P: Reactive oxygen species downregulate glucose transport system in retinal endothelial cells. Am J Physiol Cell Physiol 300: C927-C936, 2011.

11. Hart GW: Dynamic O-linked glycosylation of nuclear and cytoskeletal proteins. Annu Rev Biochem 66: 315-335, 1997.

12. Gurel Z, Sieg KM, Shallow KD, Sorenson CM and Sheibani N: Retinal O-linked N-acetylglucosamine protein modifications: implications for postnatal retinal vascularization and the pathogenesis of diabetic retinopathy. Mol Vis 19: 1047-1059, 2013.

13. Xu C, Liu G, Liu X and Wang F: O-GlcNAcylation under hypoxic conditions and its effects on the blood-retinal barrier in diabetic retinopathy. Int J Mol Med 33: 624-632, 2014.

14. Hart GW, Slawson C, Ramirez-Correa G and Lagerlof O: Cross talk between O-GlcNAcylation and phosphorylation: Roles in signaling, transcription, and chronic disease. Annu Rev Biochem 80: 825-858, 2011.

15. Zachara NE, O'Donnell N, Cheung WD, Mercer JJ, Marth JD and Hart GW: Dynamic O-GlcNAc modification of nucleocytoplasmic proteins in response to stress. A survival response of mammalian cells. J Biol Chem 279: 30133-30142, 2004.

16. Ngoh GA, Facundo HT, Zafir A and Jones SP: O-GlcNAc signaling in the cardiovascular system. Circ Res 107: 171-185, 2010.

17. Zafir A, Readnower R, Long BW, McCracken J, Aird A, Alvarez A, Cummins TD, Li Q, Hill BG, Bhatnagar A, et al: Protein O-GlcNAcylation is a novel cytoprotective signal in cardiac stem cells. Stem Cells 31: 765-775, 2013.

18. Johnson B, Opimba M and Bernier J: Implications of the O-GlcNAc modification in the regulation of nuclear apoptosis in T cells. Biochim Biophys Acta 1840: 191-198, 2014.

19. Nuñez G, Benedict MA, Hu Y and Inohara N: Caspases: the proteases of the apoptotic pathway. Oncogene 17: 3237-3245, 1998.

20. Kowluru RA and Chan PS: Oxidative stress and diabetic retinopathy. Exp Diabetes Res 2007: 43603, 2007.

21. Brownlee M: The pathobiology of diabetic complications: a unifying mechanism. Diabetes 54: 1615-1625, 2005.

22. Caldwell RB, Bartoli M, Behzadian MA, El-Remessy AE, Al-Shabrawey M, Platt DH, Liou GI and Caldwell RW: Vascular endothelial growth factor and diabetic retinopathy: role of oxidative stress. Curr Drug Targets 6: 511-524, 2005.

23. Kanwar M, Chan PS, Kern TS and Kowluru RA: Oxidative damage in the retinal mitochondria of diabetic mice: possible protection by superoxide dismutase. Invest Ophthalmol Vis Sci 48: 3805-3811, 2007.

24. Tarr JM, Kaul K, Chopra M, Kohner EM and Chibber R: Pathophysiology of diabetic retinopathy. ISRN Ophthalmol 2013: 343560, 2013.

25. Slawson C, Housley MP and Hart GW: O-GlcNAc cycling: how a single sugar post-translational modification is changing the way we think about signaling networks. J Cell Biochem 97: 71-83, 2006.

26. Ma J and Hart GW: Protein O-GlcNAcylation in diabetes and diabetic complications. Expert Rev Proteomics 10: 365-380, 2013. 
27. Yang X, Ongusaha PP, Miles PD, Havstad JC, Zhang F, So WV Kudlow JE, Michell RH, Olefsky JM, Field SJ and Evans RM: Phosphoinositide signalling links O-GlcNAc transferase to insulin resistance. Nature 451: 964-969, 2008.

28. Hanover JA, Krause MW and Love DC: The hexosamine signaling pathway: O-GlcNAc cycling in feast or famine. Biochim Biophys Acta 1800: 80-95, 2010.

29. Sun J, Xu Y, Sun S, Sun Y and Wang X: Intermittent high glucose enhances cell proliferation and VEGF expression in retinal endothelial cells: the role of mitochondrial reactive oxygen species. Mol Cell Biochem 343: 27-35, 2010.

30. Al-Shabrawey M and Smith S: Prediction of diabetic retinopathy: role of oxidative stress and relevance of apoptotic biomarkers. EPMA J 1: 56-72, 2010

31. Kennedy A and Frank RN: The influence of glucose concentration and hypoxia on VEGF secretion by cultured retinal cells. Curr Eye Res 36: 168-177, 2011.

32. Loukovaara S, Koivunen P, Inglés M, Escobar J, Vento $M$ and Andersson S: Elevated protein carbonyl and HIF-1 $\alpha$ levels in eyes with proliferative diabetic retinopathy. Acta Ophthalmol 92: 323-327, 2014

33. Izuta H, Matsunaga N, Shimazawa M, Sugiyama T, Ikeda T and Hara H: Proliferative diabetic retinopathy and relations among antioxidant activity, oxidative stress, and VEGF in the vitreous body. Mol Vis 16: 130-136, 2010.

34. Moley KH and Mueckler MM: Glucose transport and apoptosis. Apoptosis 5: 99-105, 2000.

35. Du XL, Edelstein D, Rossetti L, Fantus IG, Goldberg H Ziyadeh F, Wu J and Brownlee M: Hyperglycemia-induced mitochondrial superoxide overproduction activates the hexosamine pathway and induces plasminogen activator inhibitor-1 expression by increasing Sp1 glycosylation. Proc Natl Acad Sci USA 97: 12222-12226, 2000 .
36. Pang Y,Hunton DL, Bounelis P and Marchase RB: Hyperglycemia inhibits capacitative calcium entry and hypertrophy in neonatal cardiomyocytes. Diabetes 51: 3461-3467, 2002.

37. Zhao L, Yan X, Shi J, Ren F, Liu L, Sun S and Shan B: Ethanol extract of Forsythia suspensa root induces apoptosis of esophageal carcinoma cells via the mitochondrial apoptotic pathway. Mol Med Rep 11: 871-880, 2015.

38. Watanabe J, Nakamachi T, Ohtaki H, Naganuma A, Shioda S and Nakajo S: Low dose of methylmercury $(\mathrm{MeHg})$ exposure induces caspase mediated-apoptosis in cultured neural progenitor cells. J Toxicol Sci 38: 931-935, 2013.

39. Housley MP, Udeshi ND, Rodgers JT, Shabanowitz J, Puigserver P, Hunt DF and Hart GW: A PGC-1alpha-O-GlcNAc transferase complex regulates FoxO transcription factor activity in response to glucose. J Biol Chem 284: 5148-5157, 2009.

40. O'Rourke B: Mitochondrial ion channels. Annu Rev Physiol 69: 19-49, 2007.

41. Feissner RF, Skalska J, Gaum WE and Sheu SS: Crosstalk signaling between mitochondrial $\mathrm{Ca}^{2+}$ and ROS. Front Biosci (Landmark Ed) 14: 1197-1218, 2009.

42. Nagy T, Champattanachai V, Marchase RB and Chatham JC: Glucosamine inhibits angiotensin II-induced cytoplasmic $\mathrm{Ca}^{2+}$ elevation in neonatal cardiomyocytes via protein-associated O-linked N-acetylglucosamine. Am J Physiol Cell Physiol 290: C57-C65, 2006.

43. Ngoh GA, Watson LJ, Facundo HT and Jones SP: Augmented O-GlcNAc signaling attenuates oxidative stress and calcium overload in cardiomyocytes. Amino Acids 40: 895-911, 2011. 